\title{
Spanien: Massenmobilisierungen gegen das Austeritätsdiktat
}

Die Hintergründe der tiefen wirtschaftlichen Rezession in Spanien und ihre sozialen Folgen liefern die Folie, vor der ein Überblick über die Situation der Gewerkschaften, die massenhaften Protest- und Streikaktionen und ihre vielfältigen Akteure erst verstanden werden können. Diese Protestwelle droht nach drei intensiven Jahren 2010 - 2012 zu ermüden und trifft zuletzt auf eine neokonservative Gegenoffensive der Regierung Rajoy. Daraus ergibt sich aktuell ein strategisches Dilemma der spanischen Gewerkschaften zwischen der Einbindung in die teils delegitimierten Institutionen aus der Zeit vor der Krise und einer glaubwürdigen Beteiligung an der Protestbewegung.

HOLM-DETLEV KÖHLER, JOSÉ PABLO CALLEJA JIMÉNEZ

\section{Die wirtschaftliche Strukturkrise}

Spanien gehört mit seinen südeuropäischen Nachbarn Griechenland, Italien und Portugal sowie Irland zu den EuroLändern, die am härtesten und nachhaltigsten von der internationalen Wirtschaftskrise seit 2008 getroffen wurden. In diesen Ländern traf die internationale Kredit- und Bankenkrise auf lange aufgestaute Strukturprobleme und ließ im spanischen Fall eine aufgeblähte Immobilienblase jäh zerplatzen. Die bis Ende 2011 regierende sozialistische PSOE-Regierung (Partido Socialista Obrero Español) versuchte zunächst mit einem öffentlichen Konjunkturprogramm gegenzusteuern, sah sich jedoch Mitte 2010 angesichts der tief greifenden Folgen des wirtschaftlichen Einbruchs und der ansteigenden Staatsverschuldung gezwungen, auf den von der Troika (Europäische Kommission, Europäische Zentralbank (EZB) und Internationaler Währungsfonds (IWF)) empfohlenen Austeritätskurs umzuschwenken. Seither leidet das Land unter einer sich gegenseitig verstärkenden Spirale aus Kürzungen der öffentlichen Ausgaben und Sozialleistungen, sinkenden Löhnen und Konsumausgaben, Rekordarbeitslosigkeit, dramatischer Jugendarbeitslosigkeit, wachsender Armut und sozialer Ungleichheit, Steuererhöhungen bei sinkenden Einnahmen und weiter ansteigender Staatsverschuldung.

Selbst wenn die Löhne, Sozialabgaben und Unternehmenssteuern weiter sinken und auch die Zinsen ihren Risi- kozuschlag abbauen, wenn also alle neoliberalen Wettbewerbsbedingungen erfüllt sind, wird die spanische Wirtschaft kaum wieder ein beschäftigungswirksames dauerhaftes Wachstum erfahren. Ein Blick auf die jüngere spanische Wirtschaftsgeschichte zeigt, dass nur extern ausgelöste Sonderkonjunkturen nachhaltiges Wirtschaftswachstum erzeugen konnten, während der Normalzustand Stagnation mit Unterbeschäftigung und Emigration war. So wuchs die Wirtschaft während der Kriegskonjunktur des Ersten Weltkrieges, der technokratischen Wende des Franco-Regimes Ende der 1950er Jahre, des Beitritts zur Europäischen Gemeinschaft Mitte der 1980er Jahre und des Immobilienbooms 1994-2007. Derartige Sonderbedingungen sind derzeit nicht in Sicht und ein Spezialisierungsprofil, welches im Tourismus und Baugewerbe sowie dem kreditfinanzierten Konsum seine Wachstumsmotoren hatte, hat auch bei massiven Kostensenkungen keine Zukunft.

Die „diabolische Endlosschleife“ (Ontiveros 2013, S. 11) der Krise wird zudem durch drei sich gegenseitig verstärkende Strukturschwächen in Gang gehalten. Die enorme private Verschuldung kann bei sinkenden Einkommen und Massenarbeitslosigkeit nur sehr langsam abgebaut werden. Das durch die Finanz- und Immobilienkrise angeschlagene Bankensystem (vor allem die zwangsweise in Privatbanken umgewandelten Sparkassen) wird durch ausfallende Kredite, den Wertverfall der Immobilienanlagen und marode Hypotheken weiter geschwächt und der private Konsum ist seit Beginn der Krise drastisch eingebrochen. 
TABELLE 1

\section{Strukturdaten des spanischen Arbeitsmarktes}

Angaben in Prozent

\begin{tabular}{lc|c|c|c|c|c|c|c|c|c}
\hline & $\mathbf{1 9 9 0}$ & $\mathbf{1 9 9 2}$ & $\mathbf{1 9 9 6}$ & $\mathbf{2 0 0 0}$ & $\mathbf{2 0 0 2}$ & $\mathbf{2 0 0 7}$ & $\mathbf{2 0 0 9}$ & $\mathbf{2 0 1 1}$ & $\mathbf{2 0 1 3}$ & $\begin{array}{c}\mathbf{2 0 1 4} \\
\text { 1. Quartal }\end{array}$ \\
\hline Arbeitslosenquote & 16,3 & 18,4 & 22,2 & 14,2 & 11,4 & 8,2 & 17,8 & 21,3 & 26,0 & 25,9 \\
\hline Anteil Langzeitarbeitsloser & 51,4 & 46,6 & 54,6 & 44,6 & 33,7 & 23,7 & 28,4 & 48,1 & 58,4 & 61,7 \\
\hline Frauenarbeitslosenquote & 24,2 & 25,5 & 29,6 & 20,5 & 16,1 & 10,6 & 18,1 & 21,8 & 26,6 & 26,5 \\
\hline Jugendarbeitslosenquote & 33,0 & 35,7 & 42,0 & 28,1 & 22,1 & 18,0 & 37,7 & 46,1 & 55,4 & 55,4 \\
\hline Anteil befristeter Arbeitsverträge & 30,3 & 33,5 & 33,8 & 32,9 & 25,9 & 26,0 & 20,9 & 21,0 & 18,9 & 19,0 \\
\hline Erwerbsquote & 49,4 & 48,8 & 49,6 & 53,3 & 54,6 & 59,2 & 60,1 & 60,3 & 60,0 & 59,4 \\
\hline Weibliche Erwerbsquote & 33,3 & 34,2 & 37,2 & 39,8 & 42,7 & 49,5 & 52,0 & 53,3 & 53,9 & 53,7 \\
\hline
\end{tabular}

Quelle: Spanisches Statistisches Bundesamt INE, Erwerbsbevölkerungsumfrage.

Um aus der Wirtschaftskrise herauszufinden, müsste Spanien gleichzeitig seine öffentlichen Schulden in den Griff bekommen, die immensen privaten Schulden der Unternehmen und Haushalte abbauen und ein koordiniertes massives Investitionsprogramm in neue zukunftsträchtige Wirtschaftszweige in Gang setzen. Die drastische Austeritätspolitik seit 2010 hat die Strukturprobleme der spanischen Wirtschaft in keiner Hinsicht verbessert, sondern nur die Kosten der Krise sozial ungerecht verteilt. Die offen zutage tretende soziale Ungerechtigkeit des politischen Krisenmanagements bildet den Kontext für die strategischen Probleme und Dilemmata der Gewerkschaften. Und sie ist der Hintergrund für den Unmut weiter Bevölkerungsteile, der sich in neuen Protestformen und Bewegungen äußert.

\section{Die Folgen der Krise für Arbeits- markt und Gesellschaft}

War Spanien von 1994-2007 das europäische Land mit dem höchsten Beschäftigungswachstum, so wurde es in der Krise zum rasantesten Arbeitsplatzvernichter (Tabelle 1). Spanien entwickelte sich rasch zum europäischen Spitzenreiter in der Arbeitslosenrate und der Jugendarbeitslosigkeit. 5,9 Mio. Menschen sind laut Erwerbsbevölkerungsumfrage zu Beginn des Jahres 2014 ohne Beschäftigung. Das durchschnittliche Haushaltseinkommen sank in den Jahren 2008 - 2013 um 9,5\% infolge gekürzter Sozialleistungen und Gehälter sowie mehrerer Steuererhöhungen.

Neben dem dramatischen wirtschaftlichen Niedergang leidet die spanische Gesellschaft unter den sozialen Verwerfungen, die mit den ungerecht verteilten Kosten der Krise einhergehen. Als Folge der Massenarbeitslosigkeit sind soziale Ungleichheit und Armut stark angestiegen. Laut Cáritas Espa- ñola (2013) lebten 3 Mio. Menschen in extremer Armut (mit weniger als $307 € /$ Monat) und der armuts- und ausgrenzungsgefährdete Anteil der Bevölkerung ist von 24,1 \% (2007) auf 28,2 \% (2013) gestiegen. Laut Erwerbsbevölkerungsumfrage waren 2013 in $11 \%$ aller Haushalte (1,9 Mio.) alle erwachsenen Mitglieder arbeitslos und die stetig steigende Dauerarbeitslosigkeit hinterlässt immer mehr Menschen ohne Arbeitslosenunterstützung. Über $30 \%$ der Erwerbslosen (2,2 Mio.) waren im ersten Quartal 2013 schon länger als zwei Jahre arbeitslos und damit ohne Recht auf Arbeitslosengeld.

Spanien ist das Land der Eurozone, in dem die Einkommensungleichheit im Verlauf der Krise am stärksten gestiegen ist, sodass es heute an der Spitze der Ungleichheit in der Eurozone und an dritter Stelle in der Europäischen Union (EU) steht (Abbildung 1). Die extrem hohe Jugend-

ABB. 1

\section{Einkommensungleichheit in ausgewählten europäischen Ländern}

Angaben als Quantilsverhältnis 80/201

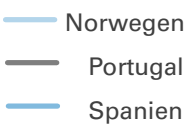

Deutschland

Spanien

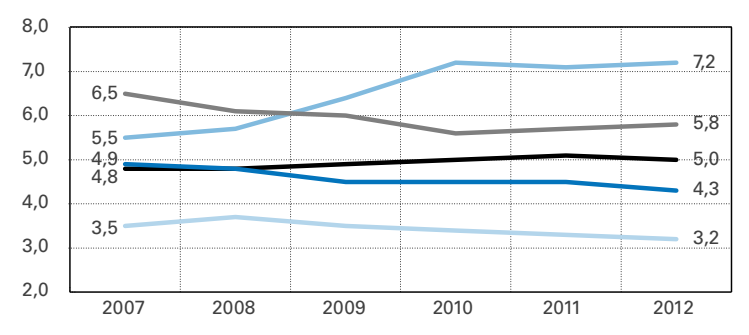

1) Das Quantilverhältnis $80 / 20$ misst den Anteil derTop- $20 \%$ Einkommensbezieher im Verhältnis zu den $20 \%$ mit dem geringsten Einkommen in der Bevölkerung.

Quelle: Eurostat; Darstellung der Autoren. 
TABELLE 2

\section{Mitgliedschaft und Organisationsgrad spanischer Gewerkschaften}

Angaben in absoluten Zahlen und in Prozent

\begin{tabular}{|c|c|c|c|c|c|}
\hline Jahr & Mitglieder & $\begin{array}{l}\text { Veränderung } \\
\qquad \text { (in \%) }\end{array}$ & Abhängig Beschäftigte & $\begin{array}{l}\text { Veränderung } \\
\quad \text { (in \%) }\end{array}$ & $\begin{array}{c}\text { Organisationsgrad } \\
\text { (in \%) }\end{array}$ \\
\hline 1977 & 1.606 .600 & - & 8.705 .200 & - & 18,4 \\
\hline 1980 & 1.109 .600 & $-30,7$ & 8.065 .600 & $-7,3$ & 13,7 \\
\hline 1985 & 1.037 .000 & $-6,5$ & 7.309 .200 & $-9,4$ & 14,2 \\
\hline 1990 & 1.561 .200 & 50,5 & 9.273 .400 & 26,8 & 16,8 \\
\hline 1995 & 1.838 .600 & 17,7 & 8.942 .700 & $-3,4$ & 20,5 \\
\hline 2000 & 2.093 .500 & 13,8 & 12.285 .700 & 37,4 & 17,0 \\
\hline 2005 & 2.700 .000 & 28,9 & 15.440 .100 & 26,6 & 17,5 \\
\hline 2010 & 2.364 .412 & $-14,1$ & 14.417 .150 & $-7,0$ & 16,4 \\
\hline
\end{tabular}

Anmerkung: Die Umfrage zur Qualität des Arbeitslebens wurde vom Spanischen Statistischen Bundesamt seit 1999 jährlich durchgeführt und ist die einzige zuverlässige Datenquelle zu gewerkschaftlichen Themen wie Organisationsgrad und Mitgliederstruktur. 2011 wurde sie im Rahmen der drastischen Sparmaßnahmen eingestellt, sodass seither keine Daten mehr verfügbar sind.

Quelle: Berechnungen der Autoren mit Daten der Sozialversicherung und des Spanischen Statistischen Bundesamtes: Umfrage zur Qualität des Arbeitslebens.

arbeitslosigkeit zeichnet dazu ein düsteres Zukunftsbild (vgl. Fundación FOESSA 2014).

Viele Haushalte leben nach dem Auslaufen des Arbeitslosengeldes von einer minimalen Armenunterstützung und sind zudem von der Zwangsräumung ihrer Wohnungen bedroht, da sie die Hypothek nicht mehr bedienen können. Seit 2009 wurden Tausende Familien ihrer Wohnungen enteignet und die Banken wurden zu den größten Immobilieneigentümern des Landes. ${ }^{1}$ Immer mehr Familien müssen ihre Wohnungen aufgeben und ziehen zu den Großeltern, deren Rente die ganze Familie unterhalten muss (sogenannte dreischichtige „Sandwich-Familien“). Ältere Menschen werden aus den Altersheimen zurück zu ihren Familien geholt, da ihre Rente die einzige Einkommensquelle ist. Ca. $8 \%$ aller arbeitslosen Haushalte leben von der Altersrente eines Mitgliedes (Laparra/Pérez Laránsus 2012, S. 131). Im Winter 2012/13 waren nach Angaben des Instituts für Umweltstudien 1,4 Mio. Haushalte ohne Strom als Folge nicht bezahlter Rechnungen. ${ }^{2}$ In dem unterentwickelten und von Haushaltskürzungen zusammengeschrumpften spanischen Sozialstaat lastet die Krise vor allem auf den Familien, die sich nicht nur materiell, sondern auch emotional immer stärker überfordert fühlen.

\section{Die Situation der Gewerkschaften}

Die spanischen Gewerkschaften werden traditionell dem lateinischen Gewerkschaftsmodell zugeordnet, welches durch niedrige Organisationsgrade, häufige Mobilisierungs- und Streikaktivitäten, fragmentierte politische Richtungsgewerkschaften, konkurrierende Tarifverhandlungsebenen und einen hohen Grad an staatlicher Intervention in die Arbeitsbeziehungen charakterisiert ist. Zwei große Gewerkschaftsbünde mit jeweils knapp einer Million Mitgliedern dominieren die Gewerkschaftslandschaft: die früher kommunistisch ausgerichteten Comisiones Obreras (CCOO) und die sozialistische Unión General de Trabajadores (UGT). In einigen Regionen mit starken nationalen Autonomiebewegungen wie dem Baskenland und Galizien konkurrieren diese mit regionalen Gewerkschaften um die Vorherrschaft. Im öffentlichen Dienst und einigen Berufszweigen (Krankenpflege, Erziehung, Fluglotsen, Sparkassen) agieren starke berufsständische Verbände (ausführlicher Köhler/Calleja Jiménez 2013).

1 Es gibt zwar keine zuverlässigen statistischen Quellen über die Anzahl der Zwangsräumungen seit dem Ausbruch der Krise, aber die spanische Zentralbank ermittelt, basierend auf Informationen der spanischen Finanzinstitute, allein für das Jahr 201232.490 Enteignungen aufgrund nicht bedienter Hypotheken (Nota informativa del 10/5/2013, www.bde.es/SalaPrensa/NotasInformativas). Für das Jahr 2013 hat der Oberste Rat für Justiz erstmals Statistiken über Zwangsräumungen von Wohnungen, Geschäften und Garagen veröffentlicht, die sich auf 67.189, d.h. im Durchschnitt 184 tägliche Vollstreckungen, belaufen. Die Plataforma de Afectados por la Hipoteca PAH (Plattform von Hypotheken-Opfern) gibt für den Zeitraum 2008-2012 insgesamt 171.110 Fälle an (Periódico Diagonal, 16.11.2012). Im Winter 2012/13 wurden 14 Selbstmorde aufgrund anstehender Zwangsräumungen registriert.

2 Der Verband für Umweltstudien (Asociación de Ciencias Ambientales, http://www.cienciasambientales.org.es/) hat im Anschluss an einen Bericht des Europäischen Wirtschafts- und Sozialrats (2011/C 44/09) den Begriff der Energiearmut (pobreza energética) geprägt, um die steigenden Schwierigkeiten der spanischen Haushalte, ihren Energiebedarf zu decken, zu erfassen. 
TABELLE 3

\section{Ergebnisse der Betriebskomitee-Wahlen in Spanien}

\begin{tabular}{|c|c|c|c|c|c|c|c|c|c|}
\hline Jahr & Abgeg. Stimmen & Delegierte & UGT & ccoo & USO & ELA-STV & LAB & CIG & Sonstige \\
\hline 1978 & 3.821 .839 & 193.112 & $21,69 \%$ & $34,45 \%$ & $5,56 \%$ & $0,99 \% \quad(18,9 \%)$ & & $0,55 \% \quad(22,3 \%)$ & $20,85 \%$ \\
\hline 1980 & 3.419 .914 & 164.617 & $29,27 \%$ & $30,86 \%$ & $8,68 \%$ & $2,44 \% \quad(25,6 \%)$ & $0,48 \%$ & $1,01 \% \quad(17,4 \%)$ & $11,94 \%$ \\
\hline 1982 & 2.987 .933 & 140.770 & $36,71 \%$ & $33,40 \%$ & $4,64 \%$ & $3,30 \% \quad(30,2 \%)$ & $0,68 \%$ & $1,17 \% \quad(18,9 \%)$ & $8,69 \%$ \\
\hline 1986 & 3.159 .776 & 177.484 & $40,19 \%$ & $34,27 \%$ & $3,83 \%$ & $2,92 \% \quad(34,9 \%)$ & $1,06 \% \quad(10,7 \%)$ & $1,34 \% \quad(21,29 \%)$ & $9,95 \%$ \\
\hline 1990 & 3.237 .776 & 221.886 & $43,10 \%$ & $37,60 \%$ & $3,00 \%$ & $3,2 \% \quad(37,84 \%)$ & $1,27 \% \quad(13,15 \%)$ & $1,5 \% \quad(23,4 \%)$ & $9,70 \%$ \\
\hline 1995 & 5.049 .334 & 219.554 & $35,51 \%$ & $37,74 \%$ & $3,56 \%$ & $2,97 \% \quad(39,73 \%)$ & $1,22 \% \quad(15,42 \%)$ & $1,91 \%$ & $17,09 \%$ \\
\hline 1999 & 5.266 .089 & 255.713 & $37,17 \%$ & $37,63 \%$ & $3,49 \%$ & $3,06 \% \quad(40,59 \%)$ & $1,33 \% \quad(15,2 \%)$ & $1,62 \% \quad(26,2 \%)$ & $15,62 \%$ \\
\hline 2003 & 6.536 .668 & 284.984 & $36,80 \%$ & $38,74 \%$ & $3,11 \%$ & $3,24 \%$ & $1,37 \% \quad(15,24 \%)$ & $1,62 \% \quad(26,2 \%)$ & $15,12 \%$ \\
\hline 2007 & 7.170 .898 & 314.174 & $37,15 \%$ & $39,09 \%$ & $2,95 \%$ & $3,13 \% \quad(40,2 \%)$ & $1,39 \% \quad(16,02 \%)$ & $1,82 \% \quad(28,6 \%)$ & $14,45 \%$ \\
\hline 2011 & 7.252 .121 & 304.845 & $36,33 \%$ & $38,38 \%$ & $3,43 \%$ & $3,03 \% \quad(39,8 \%)$ & $1,39 \% \quad(17,26 \%)$ & $1,63 \% \quad(26,48 \%)$ & $15,77 \%$ \\
\hline
\end{tabular}

Anmerkung: Neben Unión General de Trabajadores (UGT) und Comisiones Obreras (CCOO) existieren u. a. die regionalen Gewerkschaften Eusko Langileen Alkartasuna - Solidaridad de Trabajadores Vascos (ELA-STV) und Langile Abertzalen Batzordeak (LAB) im Baskenland sowie Confederación Intersindical Galego (CIG) in Galizien. Die Zahlen in Klammern beziehen sich auf den Anteil der Delegierten, den die regionalen Gewerkschaften in ihren jeweiligen Regionen erreichten. Hinter der Kategorie „Sonstige“ verbergen sich vor allem die berufsständischen Verbände der Beamten, Lehrer, Krankenpflege sowie im Einzelhandel zwei unternehmergesteuerte Verbände.

Quelle: CCOO (2012).

Die spanischen Gewerkschaften hatten sich in den zwei Jahrzehnten vor der Krise als anerkannte Verhandlungspartner in Betrieb und Politik etabliert. Bei einem stabilen Organisationsgrad um $17 \%$ der abhängig Beschäftigten waren die absoluten Mitgliedszahlen im Beschäftigungsboom stark angestiegen (Tabelle 2). CCOO und UGT sind in einem stabilen Aktionsbündnis vereint. Seit den 1990er Jahren hat sich in Spanien ein weitreichendes System institutioneller Beteiligung der Sozialpartner in Einrichtungen wie den Arbeitsämtern, der Sozialversicherung, der beruflichen Weiterbildung, den Universitäten, den Wirtschafts- und Sozialräten etc. entwickelt. Die Gewerkschaften sind dadurch auf allen Ebenen (national, regional, lokal, sektoral) in vielfältige politische Verhandlungsprozesse eingebunden. Das Klima des sozialen Dialogs führte zu zahlreichen tripartistischen Sozialpakten (Konzertierung) über Wirtschafts- und Beschäftigungspolitik, Rentenreform, Gesundheitssystem und sozialen Dialog im öffentlichen Dienst. Die Folgen der aktuellen Wirtschaftskrise mit den drastischen Austeritätsprogrammen und zwei gewerkschaftsfeindlichen Arbeitsmarktreformen (2010, 2012) haben den sozialen Dialog auf vielen Ebenen zum Erliegen gebracht. Es ist derzeit unsicher, ob und wann er wiederbelebt werden kann. Konzertierung und sozialer Dialog erwiesen sich erfolgreich bei der Verteilung wachsender öffentlicher Ressourcen, scheitern jedoch beim Ziel einer sozial gerechten Verteilung der Krisenfolgen.

Das Gesetz zur Gewerkschaftsfreiheit (1985) etablierte ein allgemeines Repräsentativitätskriterium, nach dem Gewerkschaften, die bei den Betriebskommitee-Wahlen landesweit über $10 \%$ oder in einer Region ${ }^{3}$ über $15 \%$ der Delegierten erzielen (zu Wahlergebnissen und nationalen wie regionalen Gewerkschaften vgl. Tabelle 3), ein generelles Recht zur Vereinbarung von Tarifverträgen besitzen, in den öffentlichen Institutionen vertretungsberechtigt sind und Zugang zu bestimmten staatlichen Subventionen haben.

Die Tarifbindung ist in Spanien generell hoch (bei ca. $80 \%$ aller Beschäftigten) aufgrund der automatischen Allgemeinverbindlichregel (erga omnes), nach der alle Betriebe unabhängig von der Mitgliedschaft unter die Regel des gültigen Branchen-Tarifvertrages fallen.

Die starke institutionelle Einbindung der Gewerkschaften in tripartistische soziale Dialoge und staatliche Institutionen gibt den Gewerkschaften zwar Einfluss, Gehör und Zugang zu organisatorischen Ressourcen, doch ist sie für das Image und öffentliche Ansehen der Arbeitnehmerorganisationen eher schädlich. Als Teil des politischen Systems erleiden sie denselben Vertrauensverlust wie die Politik und bilden zusammen mit den politischen Parteien, Banken und multinationalen Unternehmen die Institutionen-Gruppe des geringsten Vertrauens in der spanischen Gesellschaft. ${ }^{4}$

Das Streikrecht ist in Spanien ein in der Verfassung verankertes kollektiv ausgeübtes individuelles Grundrecht und nicht wie in einigen nordeuropäischen Ländern an gewerkschaftliche Organisationen und Urabstimmungen gebunden. Aussperrungen sind dagegen gesetzlich stark eingeschränkt auf Ausnahmesituationen.

Ähnlich wie andere südländische Gewerkschaften gelten die spanischen als relativ streikfreudig und die Mobilisie-

3 Der spanische Staat besteht aus 17 Regionen (Comunidades Autónomas)

4 Siehe dazu die regelmäßigen Meinungsumfragen von Metroscopia (www.metroscopia.org) und des Zentrums für Soziologische Forschung CIS (www.cis.es). 
rungsfähigkeit übersteigt den Grad der Mitgliedschaft. Gleichwohl lässt sich auch in Spanien ein langfristiger Trend zu nachlassenden Konflikten und sinkender Streikhäufigkeit feststellen (Luque Balbona 2012, 2013). Die Streikentwicklung in Spanien folgt einer stark politisch motivierten Dynamik mit weniger Konflikten in Phasen intensiver Konzertierung und guten Beziehungen zwischen den Sozialpartnern und zwischen Gewerkschaften und Regierung, wie z.B. in den Jahren der sozialistischen Regierung Zapatero vor dem Ausbruch der Krise (2005 - 2009) und ansteigender Streikhäufigkeit in Phasen politischer Unzufriedenheit seitens der Gewerkschaften (Abbildung 2).

Streiks und Arbeitskonflikte folgen in Spanien einigen längerfristigen Trends, die auch in anderen südeuropäischen Ländern beobachtbar sind. Neben der sinkenden Streikhäufigkeit kommt es zu einem Wandel der Inhalte und Adressaten. Während strikte ökonomische Streiks um Löhne und Arbeitsbedingungen im privaten Sektor stark zurückgehen, nehmen politisch motivierte Streiks zu. Adressaten der Protestaktionen sind weniger die Arbeitgeber als die politisch Verantwortlichen. Dieser Trend wird auch dadurch verstärkt, dass bei vielen Konflikten um Werksschließungen und Produktionsverlagerungen die unternehmerischen Entscheidungsträger in ausländischen Konzernzentralen sitzen und für die Betroffenen nicht greifbar sind. Viele Probleme des Arbeitsmarktes wie die zunehmende Prekarisierung, die Fragmentierung der Belegschaften und der verschärfte Wettbewerbsdruck nehmen zudem einen systemischen Charakter an und sind kaum einzelnen Unternehmern anzulasten.

Ein weiterer längerfristiger Trend in der Entwicklung der Arbeitskonflikte ist ihr Defensivcharakter. Die Arbeitnehmer und ihre Gewerkschaften kämpfen nicht mehr für die Verbesserung ihrer Arbeitsbedingungen und die Ausweitung ihrer Rechte, sondern um den Erhalt ihrer Arbeitsplätze, gegen den Abbau des Kündigungsschutzes, die Verlängerung der Arbeitszeiten, die Verlagerung der Produktionskapazitäten oder die Prekarisierung der Arbeitsverträge. Dies deutet auf eine strategische Schwäche der Gewerkschaften und der spanischen Linken insgesamt hin.

\section{Massenmobilisierungen gegen das Austeritätsdiktat}

Seit dem definitiven neoliberalen Schwenk der sozialistischen Regierung im Mai 2010 kam es zu einer ansteigenden Protestwelle gegen die sozialen Kürzungen, die Beschneidung der Arbeitnehmerrechte, die zunehmenden sozialen Verwerfungen der Krise und gegen eine generelle Degradierung der Zivilgesellschaft. Zuerst protestierten die Arbeitnehmer und ihre Gewerkschaften mit einem Generalstreik (29.09.2010) gegen die Arbeitsmarkt- und Sozialreformen sowie den neuen Schuldendeckel der Verfassungsreform. Das Jahr 2011 erlebte einen Höhepunkt der sozialen Protestwelle mit der von
ABB. 2

\section{Streikbedingt ausgefallene Arbeitstage in Spanien und Deutschland}

Angaben in absoluten Zahlen

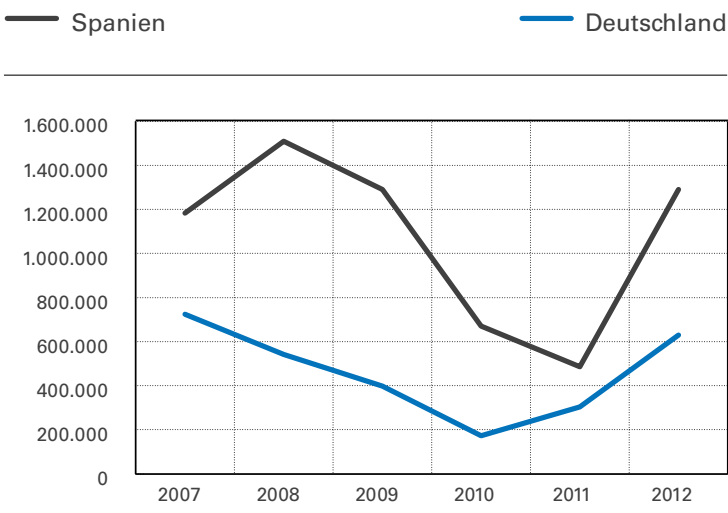

Quelle: WSI, Spanisches Ministerium für Arbeit und Soziales; Darstellung der Autoren.

der arbeits- und perspektivlosen Jugend getragenen Bewegung 15-M. Gleichzeitig kam es zu Streiks vor allem im öffentlichen Dienst. Unter dem verschärften Austeritätsdiktat der neuen konservativen Volksparteiregierung ereigneten sich 2012 andauernde Massenproteste vieler sozialer Gruppen und zwei weitere Generalstreiks der Gewerkschaften am 29.03.2012 und 14.11.2012. Letzterer erfolgte zeitgleich mit dem europäischen Protesttag des Europäischen Gewerkschaftsbundes. Insbesondere die Beschäftigten des öffentlichen Dienstes befinden sich in einem permanenten Abwehrkampf gegen immer neue Kürzungen und Eingriffe in angestammte Rechte und Bedingungen, während die anwachsenden Schichten sozial bedrohter Bevölkerungsgruppen unter dem Niedergang sozialer Leistungen, dem Verfall des Bildungs- und Gesundheitswesens und den leeren Sozialkassen leiden.

Der Unmut in der Bevölkerung über das inkompetente und sozial unausgewogene Krisenmanagement der Regierungen und die anhaltende Welle von Korruptionsskandalen auf allen politischen Ebenen führt zu einer anwachsenden Distanz gegenüber der politischen Klasse, aber auch zu neuen zivilen Protestbewegungen. Allen gängigen Umfragen zufolge ist die politische Klasse Spaniens die am deutlichsten diskreditierte soziale Gruppe und die Korruption von Politik und Verwaltung ist inzwischen in der Wahrnehmung der Bevölkerung das drängendste Problem neben der Arbeitslosigkeit.

Der wachsende Unmut über die soziale und politische Situation führte zur Bildung vieler ziviler Oppositionsgruppen insbesondere unter den Jugendlichen, die sich um ihre Zukunftsperspektiven betrogen fühlen. Das Internet und die virtuellen Netzwerke (Facebook, Twitter, etc.) boten für diese sehr heterogenen Gruppen eine agile Kommunikationsplattform. Spontane Bewegungen in anderen Ländern (z. B. der ,arabische Frühling“) waren eine zusätzliche Inspi- 
TABELLE 4

\section{Motiv und Gefolgschaft der Generalstreiks}

Angaben in absoluten Zahlen und in Prozent

\begin{tabular}{ll|c|c|c}
\hline Datum & Anlass & $\begin{array}{c}\text { Teilnehmer } \\
\text { (inTsd.) }\end{array}$ & $\begin{array}{c}\text { Abh. Beschäftigte } \\
\text { (inTsd.) }\end{array}$ & $\begin{array}{c}\text { Streikbeteiligung } \\
\text { (in \%) }\end{array}$ \\
\hline 29.09 .2010 & $\begin{array}{l}\text { Arbeitsmarktreform der } \\
\text { sozialistischen Regierung }\end{array}$ & $2.148,5$ & $15.346,8$ & 14,0 \\
\hline 29.03 .2012 & $\begin{array}{l}\text { Arbeitsmarktreform der } \\
\text { Volkspartei-Regierung }\end{array}$ & $3.357,3$ & $14.347,2$ & 23,4 \\
\hline 14.11 .2012 & $\begin{array}{l}\text { Austeritätspolitik und } \\
\text { soziale Kürzungen }\end{array}$ & $3.070,3$ & $14.347,2$ & 21,0 \\
\hline
\end{tabular}

Quelle: Berechnungen der Autoren auf Basis der Barometer des Zentrums für Soziologische Forschung (CIS) und der Erwerbsbevölkerungsumfrage.
Land organisiert und ein breites Aktionsbündnis gegen den Sozialabbau organisiert (den sog. Sozialen Gipfel mit ca. 150 Organisationen).

Der öffentliche Dienst, dessen Beschäftigte ganz unmittelbar von Sozialabbau, Gehaltskürzungen und Arbeitszeitverlängerungen betroffen sind, übernahm eine Führungsrolle bei den Massenprotesten, die in den einzelnen Regionen unterschiedliche Intensität erreichten. So organisierten die Beschäftigten des Gesundheitswesens eine weiße (marea blanca), des Sozialwesens eine orangene (marea naranja), die des Bildungswesens eine grüne (marea verde), die wissenschaftlichen Arbeiter eine rote (marea roja) und die Verwaltungen eine schwarze Protestwelle (marea negra). Immerhin ist es der weißen Welle in Madrid gelungen, das Projekt der Privatisierung der Krankenhäuser zu Fall zu bringen.

Im privaten Sektor kam es zu unterschiedlichen Reaktionen. Während sich viele von Schließungen (Werften, Bergbau) oder massivem Personalabbau (Banken und Sparkassen) bedrohte Belegschaften sehr aktiv an den Massenprotesten beteiligten und einige spektakuläre Aktionen wie den schwarzen Marsch der Bergarbeiter nach Madrid im Sommer $2012^{6}$ organisierten, verfolgten andere, wie die Werke der großen Automobilkonzerne, eine Strategie der Konzessionsverhandlungen, um dadurch im internationalen konzerninternen Standortwettbewerb neue Modelle zu bekommen und ihre Arbeitsplätze (häufig auf Kosten anderer Werke in anderen Ländern) zu sichern (Aguilar 2013).

Aus Sicht der Gewerkschaften war neben den sozialen Einschnitten insbesondere eine erneute Arbeitsmarktreform Anlass zu Kritik und Gegenwehr. Dabei handelt es sich um eine getreue Umsetzung der von der EZB geforderten Maßnahmen unter Ausschluss von Gewerkschaften und Arbeitgebern. ${ }^{7}$ Die wichtigsten Maßnahmen des Gesetzes zur Reform des Arbeitsmarktes vom 10.2.2012 (RD-Ley 3/2012):

5 Die Bewegung wurde zuerst unter Bezug auf Hessel (2010) "Los indignados" genannt, doch mit der Zeit bürgerte sich die Referenz auf das Datum des ersten Protesttages 15-M ein.

Auf der anderen Seite zeigen das Abflauen der Bewegung im Gefolge des konservativen Wahlsieges seit Ende 2011 und die insgesamt mageren Erfolge auch klar die Grenzen dieser sozialen Bewegungsformen auf. So konnten die „Marchas de la Dignidad“ (Märsche für Menschenwürde) im März 2014 zwar mehr als 50.000 Teilnehmer aus allen Teilen Spaniens zu einem Protesttag in Madrid mobilisieren, wurden jedoch von Politik und Medien ignoriert.

\subsection{Die Rolle der Gewerkschaften in der sozialen Protestwelle}

Die spanischen Gewerkschaften wurden durch den politischen Richtungswechsel seit 2010 und die Aufkündigung des impliziten Sozialpakts seitens der Arbeitgeber geradezu in die Rolle einer außerparlamentarischen Opposition gedrängt. Seither haben sie zu drei Generalstreiks aufgerufen (Tabelle 4), zahlreiche Massendemonstrationen im ganzen
6 Am 10. Juli 2012 um Mitternacht trafen Hunderte von Bergarbeitern mit leuchtenden Lampen an den Helmen, umjubelt von Tausenden solidarischer Demonstranten, nach mehrwöchigen Märschen aus den Hunderte Kilometer entfernten Kohlerevieren in der Hauptstadt ein, um gegen die drastischen Kürzungen der Kohlebeihilfen zu protestieren, die praktisch die Schließung aller Zechen bedeuten.

7 Die Hegemonie neoliberaler Politik in der EU wird in den Briefen des ehemaligen EZB-Präsidenten Trichet an die Regierungen Italiens und Spaniens im Sommer 2011 unmittelbar deutlich. Die Finanzkrise wird darin nicht als Ergebnis deregulierer Finanzmärkte, Immobilienblasen oder sonstiger wirtschafts- und finanzmarktinduzierter Probleme interpretiert, sondern den hohen Lohnkosten, dem Kündigungsschutz, flächendeckenden Tarifverhandlungen und der Arbeitslosenunterstützung angelastet. Entsprechend werden der Ankauf von Staatsanleihen und ähnliche finanzielle Unterstützungen an die Durchführung neoliberaler Arbeitsmarktreformen gebunden. 
- drastische Reduzierung des Kündigungsschutzes: Abfindungszahlungen werden auf 33 Arbeitstage pro geleistetes Beschäftigungsjahr und maximal 24 Monatsgehälter gekürzt (bisher waren es 42 Arbeitstage und höchstens 42 Monatsgehälter). Diese Abfindungshöhe wird jedoch in Zukunft Ausnahme bleiben, da bei gerechtfertigten Kündigungen nur noch 20 Arbeitstage und maximal ein Jahresgehalt gezahlt werden müssen und Rechtfertigungen für Kündigungen deutlich vereinfacht wurden. Schon vorübergehende Verluste oder erwartete zukünftige Absatzrückgänge erlauben Entlassungen.

- Kollektive Entlassungen erfordern keine staatliche Genehmigung mehr und müssen nicht mehr mit dem Betriebskomitee bzw. den Gewerkschaftsvertretungen verhandelt werden.

- Arbeitgeber können zudem ohne Verhandlung mit dem Betriebsrat aus der Tarifbindung ausscheren und Lohnkürzungen oder Arbeitszeitverlängerungen anordnen, sofern dafür „ökonomische, technische oder wettbewerbliche“ Gründe angegeben werden.

- Den Tarifverträgen auf Unternehmensebene wird ausdrücklich Vorrang vor überbetrieblichen Tarifverträgen eingeräumt.

- Die automatische Verlängerung bestehender Tarifverträge im Falle des Scheiterns von Neuverhandlungen wird auf ein Jahr beschränkt. Danach fällt die Tarifbindung weg, sofern kein neuer Tarifvertrag abgeschlossen wurde.

Diese bisher radikalste Reform des Arbeitsrechts stärkt somit explizit die individuelle Arbeitgebermacht gegenüber kollektiven Regelungen und schwächt die Verhandlungsmacht der Gewerkschaften wie insgesamt die Tarifautonomie. Die Gewerkschaften organisierten neben dem Generalstreik mehrere nationale Protesttage in vielen Städten gegen das Reformpaket, konnten es aber weder verhindern noch korrigieren. Das offizielle Reformziel, die Förderung der Beschäftigung und deren Sicherung durch „interne Flexibilität“ (Anpassung durch Arbeitszeitflexibilität, Kurzarbeit und funktionale Mobilität ohne Entlassungen), wurde bislang klar verfehlt. Das implizite Ziel einer Machtverschiebung zugunsten des Unternehmerlagers wurde dagegen erreicht, denn die Gewerkschaften sehen sich einer Unternehmeroffensive in den Tarifverhandlungen, einer einseitigen Aufkündigung des sozialen Dialogs und billigen Massenentlassungen ohne Gegenwehr gegenüber. Die Zahl der abgeschlossenen Tarifverträge geht seit der Reform kontinuierlich zurück und vielen Beschäftigten droht der Verlust der Tarifbindung.

Die Arbeitsmarktreform ist auch deshalb ein frontaler Angriff gegen die Tarifautonomie, weil sie kurz zuvor getroffene bilaterale Vereinbarungen zwischen Gewerkschaften und Arbeitgebern außer Kraft setzt. Wenige Wochen vor der Verabschiedung der Reform hatten die Spitzenverbände das II. Abkommen über Beschäftigung und Tarifverhandlungen 2012 - 2014 mit weitreichenden Mechanismen zur Flexibilisierung der Löhne und des Arbeitseinsatzes unterzeichnet. Dazu wurde das V. Abkommen zur autonomen Schlichtung von Arbeitskonflikten vereinbart. Diese Versuche einer Wiederbelebung des bilateralen sozialen Dialogs brach das Gesetz schlicht ab.

Wie oben angedeutet hatten sich die spanischen Mehrheitsgewerkschaften an den kontinuierlichen sozialen $\mathrm{Di}$ alog und die Einbindung in das politisch-institutionelle System gewöhnt und stehen nun vor dem Problem, mit dieser Tradition radikal zu brechen und sich wieder in eine soziale Protestbewegung zu verwandeln oder einen schwierigen Spagat zwischen Protest und Beteiligung durchzuhalten.

\subsection{Abflauen der Proteste und neokonservative Gegenoffensive}

Der offen antigewerkschaftliche Charakter der Arbeitsmarktreform steht in Zusammenhang mit einer generellen neokonservativen Offensive der Zentralregierung und der konservativen Regionalregierungen. Die gezielte Diskreditierung und Schwächung der gewerkschaftlichen Organisationen bildet zusammen mit der Kriminalisierung des sozialen Protests ein Kernelement dieser Offensive. Neben der Aushöhlung der Tarifautonomie werden dabei Subventionen an soziale Organisationen gekürzt, Freistellungsrechte von Delegierten beschnitten und gezielte antigewerkschaftliche Kampagnen gefahren. Spanien ist ein Schulbeispiel für die Instrumentalisierung der massenhaften Angst vor der Arbeitslosigkeit für einen neoliberalen Umbau der Arbeitsbeziehungen im Sinne von Lohnkürzungen, Arbeitszeitverlängerungen, Aushöhlung des Flächentarifvertrages, Schwächung der Gewerkschaften und Deregulierung des Arbeitsmarktes.

Die seit Dezember 2011 regierende Volkspartei unter Mariano Rajoy begegnet den andauernden Massenprotesten mit einem Diskurs der unvermeidlichen Einschnitte zur Überwindung der Krise und einer notwendigen Mimesis der Empfehlungen der Troika. Den Popularitätsverlust kann sie deshalb gut überstehen, da er die gesamte politische Klasse betrifft und die diskreditierte und konzeptlose sozialistische Opposition aus dem Negativimage der Regierung keinerlei politisches Kapital zu schlagen vermag. Gegenüber den protestierenden und streikenden Massen auf der Straße stellt sie sich als Vertreterin der schweigenden Mehrheit dar und findet dabei einen durchaus wirkungsvollen Partner in der katholischen Kirche.

Seit 2013 lässt sich ein möglicherweise nachhaltiger Wandel der politischen Kräfteverhältnisse in Form einer neokonservativen Gegenoffensive beobachten, der im demokratischen Spanien seit den 1970er Jahren beispiellos ist. Die zivile jugendliche Massenbewegung des 15-M hat sich weitgehend in kleine lokale Gruppen und Initiativen aufgelöst, zu denen lokale Selbsthilfegruppen, ethische Finanzinstitute, alternative Energie-Projekte, ökologische Konsum- und Landwirtschaftsgruppen, Wohnungs- 
bau-Kooperativen etc. gehören. In der Öffentlichkeit ist die Plattform gegen Zwangsräumungen von Wohnungen am sichtbarsten.

Die zunehmend zerstreute soziale Protestbewegung und die geschwächten Gewerkschaften drohen jedoch in der aktuellen Flut von antisozialen Maßnahmen und Gesetzen zu ertrinken. Eine Rentenreform bindet die jährliche Anpassung an die steigende Lebenserwartung, was für die $\mathrm{Zu}$ kunft einen dauerhaften realen Einkommensverlust der Rentenempfänger bedeutet. Mehrere Landesregierungen privatisieren das Gesundheitswesen, das Schulgeld für Kinder armer Familien wurde zusammengestrichen und die Universitätsgebühren erhöht. Eine Bildungsreform streicht das Fach Bürgererziehung und erhebt den Religionsunterricht wieder zum Pflichtfach, stärkt die Stellung der privaten katholischen gegenüber den staatlichen Schulen und ermöglicht wieder nach Geschlechtern getrennte Schulsysteme. Die Abtreibungsreform hebt die von der sozialistischen Regierung 2010 nach jahrzehntelangen Kämpfen endlich eingeführte Fristenregelung auf und stellt die Schwangerschaftsunterbrechung wieder unter Strafe.

Ein neues Sicherheitsgesetz etabliert einen umfassenden Katalog von Vergehen, die mit polizeilichen Bußgeldern ohne Notwendigkeit richterlicher Anordnung bis $\mathrm{zu}$ $30.000 €$, in schweren Fällen bis zu $600.000 €$ geahndet werden können, wozu u. a. nicht genehmigte Demonstrationen, die Beleidigung staatlicher Organe und Symbole, die Besetzung öffentlicher Plätze oder Gebäude, das Fotografieren von Sicherheitskräften (oft der einzige Beleg für Anklagen gegen deren brutales Vorgehen) u.v.m. gehören. Darüber hinaus stattet es private Wachdienste mit polizeilichen Vollmachten wie Ausweiskontrolle, Verhaftung und Leibesvisitation aus. Eine Gemeindereform schränkt die Selbstverwaltungsrechte und Finanzierungsquellen der Kommunen drastisch ein und entzieht ihnen unter anderem die Kompetenzen in der sozialen Fürsorge. Gegen alle diese und etliche weitere Maßnahmen ruft der von den Gewerkschaften geführte „Soziale Gipfel“ gemeinsam mit zahlreichen Betroffenen-Organisationen überall zu Protestaktionen und Widerstand auf, doch deren Wirkungslosigkeit scheint den neokonservativen Reformeifer nur noch weiter anzuheizen.

Wie können die Gewerkschaften in diesem Kontext wieder zu einer glaubwürdigen Bedrohung für das neoliberale Establishment werden? Eine fundamentale Herausforderung für die spanischen Gewerkschaften liegt in der Rückgewinnung ihrer sozialen Reputation und politischen Glaubwürdigkeit. Im Unterschied zu den politischen Parteien, die trotz der Verwicklung in unzählige Korruptionsskandale weiterhin gewählt werden, oder des Arbeitgeberverbandes, dessen Präsident wegen Wirtschaftskriminalität entlassen werden musste und in Haft ist, werden die Gewerkschaften durch Beteiligungen an unehrenhaften Machenschaften unmittelbar geschwächt. Die Verwicklung der andalusischen Mehrheitsgewerkschaften in den Skandal um gefälschte Vorruhestands- und Kollektiventlassungsvereinbarungen, der UGT-Skandal um gefälschte Rechnungen mit dem Ziel überhöhter öffentlicher Zuschüsse für Veranstaltungen und Bildungsmaßnahmen oder die Beteiligung der Gewerkschaftsvertreter in den Verwaltungsräten der Sparkassen, die sich in der Finanz- und Immobilienkrise blamierten, beschädigen das angeschlagene Image der Gewerkschaften weiter. Im von Korruptionsskandalen geplagten Andalusien mussten die Führungen der sozialistischen Regierungspartei PSOE, der Vorsitzende des regionalen Arbeitgeberverbandes und der Generalsekretär der sozialistischen Gewerkschaft UGT zurücktreten. Partei und Arbeitgeberverband funktionieren mit neuen Vorständen weiter wie bisher, während die UGT in einer existenziellen Finanz- und Mitgliederkrise ist, von der die gesamte spanische Gewerkschaftsbewegung betroffen ist. Gäbe es bei den Gewerkschaften nur annähernd so viele Korruptionsskandale wie bei den Arbeitgebern und politischen Parteien, wären sie längst ohne Mitglieder und Gefolgschaft.

\section{Schlussdiskussion}

Spanien hat sich seit dem Ausbruch der Krise zu einem Paradebeispiel für die tief greifenden sozialen Wandlungen im Kontext eines konsolidierten neoliberalen Hegemonieprojektes entwickelt. Das Zusammenspiel von Marktliberalismus und autoritärem Sicherheitsstaat zersetzt die Institutionen und Organisationen, die in der Vergangenheit korrigierend eingriffen, um soziale Integration und Ausgleich zu erzielen. Der Bürger in Arbeit und Gesellschaft wird dabei zum machtlosen Spielball dominanter Marktund Sicherheitskräfte degradiert.

Nach Richard Hyman (2001) agieren Gewerkschaften immer in einem strategischen Dreieck, gebildet aus Markt (Aushandeln der Bedingungen der Arbeitsverträge), Klasseninteresse (Verteidigung der Interessen der Lohnabhängigen) und Gesellschaft (zivilgesellschaftlicher Akteur eingebunden in Institutionen, Traditionen und Diskurse). Dieses komplexe Dreiecksverhältnis enthält die strategische Spannung zwischen „Ordnungsfaktor und/oder Gegenmacht“ (Schmidt 1972), zwischen Mobilisierung und Konzertierung, zwischen Konflikt und Kooperation, „boxing or dancing“ (Huzzard et al. 2005). Dabei verfügen die Gewerkschaften über vier traditionelle Machtquellen: strukturelle (Verhandlungsmacht auf den internen und externen Arbeitsmärkten), organisatorische (Mitgliedschaft und Militanz), institutionelle (offizieller Status als Sozialpartner) und kommunikative Macht (soziale Legitimität als gesellschaftlicher Akteur) (Gumbrell-McCormick/Hyman 2013; Brinkmann et al. 2008; Urban 2010).

In allen vier Machtdimensionen haben die spanischen Gewerkschaften - ebenso wie viele ihrer europäischen Partnerorganisationen - in den letzten Jahren empfindliche Verluste erlitten. Krise und Massenarbeitslosigkeit, Prekarisierung und Globalisierung haben die Verhandlungs- 
position in Betrieben und Wirtschaft geschwächt. Mitgliederverluste und ein Wandel zu mehr instrumenteller, weniger militanter Einstellung vermindern die organisatorische Stärke. Sozialabbau und Arbeitsmarktreformen minimieren die institutionelle Macht. Und die neoliberale Dominanz sowie interne Krisen und ein negatives Image haben die Legitimität der Gewerkschaften in der Gesellschaft auf einen Tiefpunkt gebracht.

$\mathrm{Ob}$ und wie es den spanischen Gewerkschaften in dieser schwierigen Lage gelingt, alte Machtressourcen zu erneuern und neue zu erschließen, hängt sicher nicht nur von ihnen selbst ab, sondern auch von sozialen Kontextbedingungen, insbesondere vom Erstarken einer Opposition gegen die neoliberale Hegemonie und auch von der Wiederbelebung des sozialen Europaprojekts. Als strategische Akteure eröffnen sich ihnen vor allem zwei Handlungsfelder: eine interne organisatorische Erneuerung und externe Allianzen mit zivilen Protestbewegungen. Der Erfolg der weißen Welle im Kampf gegen die Privatisierung der Madrider Krankenhäuser zeigt dabei einige Orientierungen. Eine zielorientierte Allianz zwischen verschiedenen, großen und kleinen Gewerkschaften und Berufsverbänden, ein Bündnis von Beschäftigtengruppen und Nutzern unter Einbeziehung ziviler Protestbewegungen und der Einsatz eines variablen Sets von Streikund Protestaktionen können einige der strategischen Schwächen der Gewerkschaften überwinden helfen.

Mit ihren 2,5 Mio. Mitgliedern, 7 Mio. Wählerstimmen bei Betriebskomitee-Wahlen und 11 Mio. Beschäftigten mit Tarifbindung bilden die spanischen Gewerkschaften weiterhin die wichtigsten sozialen Organisationen der spanischen Gesellschaft. Gleichwohl kommt es für sie darauf an, durch eine Rückkehr in die Gesellschaft wieder zu einer glaubwürdigen Drohung und Opposition für arbeitnehmerfeindliche und antisoziale Kräfte zu werden.

\section{LITERATUR}

Aguilar, S. (Hrsg.) (2013): Anuari del Conflicte Social 2012, Barcelona Brinkmann, U./Choi, H.-L./Detje, R./Dörre, K./Holst, H./Karakayali, S./ Schmalstieg, C. (2008): Strategic Unionism: Aus der Krise zur Erneuerung?, Wiesbaden

Busch, K./Hermann, C./Hinrichs, K./Schulten, T. (2012): Eurokrise, Austeritätspolitik und das Europäische Sozialmodell, Berlin

Cáritas Española (2013): VIII Informe del Observatorio de la Realidad Social, Madrid
Confederación Sindical de Comisiones Obreras (CCOO) (2012): Informe de elecciones sindicales, Madrid

Fundación Foessa (2014): Informe 2014: Precariedad y Cohesión Social, Madrid (www.foessa.es)

Gumbrell-McCormick, R./Hyman, R. (2013): Trade unions in Western Europe. Hard times, hard choices, Oxford

Hessel, S. (2010): Indignez-vous!, Paris

Huzzard, T./Gregory, D./Scott, R. (Hrsg.) (2005): Strategic unionism and partnership: Boxing or dancing?, Houndsmills

Hyman, R. (2001): Understanding European trade unionism. Between market, class \& society, London

Köhler, H.-D./Calleja Jiménez, J. P. (2013): Die Gewerkschaften in Spanien, Friedrich Ebert Stiftung, Berlin (http://library.fes.de/pdf-files/id/10133.pdf) Laparra, M./Pérez Laránsus, B. (Koord.) (2012): Crisis y fractura social en Europa, Barcelona: Fundación La Caixa: Colección Estudios Sociales Nr. 35 Luque Balbona, D. (2012): Huelgas e intercambio político en España, in: Revista Internacional de Sociología 70 (3), S. 561-585

Luque Balbona, D. (2013): La forma de las huelgas en España, 1905-2010, in: Política y Sociedad 50 (1), S. 235-268

Ontiveros, E. (2013): Eine progressive Wachstumsstrategie für Spanien, in: Dauderstädt, M./Hillebrand, E. (Hrsg.): Alternativen zur Austerität, Berlin, S. $11-14$

Schmidt E. (1972): Ordnungsfaktor oder Gegenmacht - Die politische Rolle der Gewerkschaften, Frankfurt

Urban, H.-J. (2010): Wohlfahrtsstaat und Gewerkschaftsmacht im FinanzmarktKapitalismus: Der Fall Deutschland, in: WSI Mitteilungen 63 (9), S. 443-449, http://www.boeckler.de/cps/rde/xchg/hbs/hs.xsl/wsi_25463_25474.htm

\section{AUTOREN}

HOLM-DETLEV KÖHLER, Prof. Dr., lehrt Soziologie an der Universität von Oviedo. Arbeitsschwerpunkte: vergleichende Industrielle Beziehungen, multinationale Unternehmen, Innovation und institutioneller Wandel

hkohler@uniovi.es

José PABLo CALLEJA JimÉnez, Prof., lehrt Soziologie und Soziale Arbeit an der Universität von Oviedo. Arbeitsschwerpunkte: Gewerkschaften, soziale Bewegungen, soziale Intervention

callejajose.uo@uniovi.es 